\title{
MUSIC Algorithm Without Diagonal Elements of Scattering Matrix
}

\author{
Won-Kwang Park
}

\begin{abstract}
It is well-known that diagonal elements of scattering matrix are significantly affected by not only the anomaly but also the antennas. Due to this reason, if one applies MUltiple SIgnal Classification (MUSIC) algorithm for imaging anomaly, several artifacts are also included. In this contribution, we apply MUSIC algorithm by eliminating diagonal elements of scattering matrix and examine that the result is better than the traditional one.
\end{abstract}

Keywords-scattering matrix, MUltiple SIgnal Classification (MUSIC) algorithm, diagonal elements

\section{Introduction}

One of the important purpose of inverse scattering problem is to identify the characteristics of unknown anomaly from measured scattered field scattering parameters. Throughout many researches, various techniques for identifying location or shape of small anomaly have been investigated. Most of algorithms are based on the Newton-type based iteration schemes, refer to [1-4]. However, in order to guarantee a good result, these schemes must be started with a good initial guess which is close to the unknown anomaly. To produce a good initial guess, alternative non-iterative techniques have been developed.

Among them, MUSIC algorithm has shown its feasibilities of imaging performance. Related works can be found in [5-14] and references therein. Notice that most of researches assumed that diagonal elements of scattering matrix are dependent on the existence of anomaly and independent of the dipole antennas. But, throughout experimental simulations, they are significantly affected by the antennas also (see [15] for instance). Therefore, it is natural to extend MUSIC algorithm without diagonal elements of scattering matrix. In this paper, we introduce a MUSIC-type imaging algorithm without diagonal elements of scattering matrix and exhibit simulation results with and without diagonal elements of matrix.

This paper is organized as follows. In section II, we briefly introduce the scattering parameters and apply MUSIC algorithm without diagonal elements. In section III, we present simulation results to explain that imaging performance without diagonal elements is better than that with diagonal elements because only the diagonal elements of the scattering matrix are affected by antennas, the result will be better than that with diagonal elements, and the designed approach will be useful. Finally, a brief conclusion is given in section IV.

Won-Kwang Park

Colleague of Science and Technology / Kookmin University Republic of Korea

\section{Scattering Parameters and MUSIC Algorithm}

The scattering parameter (or S-parameter) $\mathrm{V}_{\mathrm{m}}^{-} / \mathrm{V}_{\mathrm{n}}^{+}$is defined as the ratio of the output voltage (or reflected waves) at the m-th antenna and the input voltage (or incident waves) at the $n$-th antenna. We denote $S(m, n)$ be the scattered field $\mathrm{S}$-parameter obtained by subtracting the S-parameters for the total and incident fields. Following [16], by letting $\Omega$ as the region of interest $(\mathrm{ROI}), \mathrm{S}(\mathrm{m}, \mathrm{n}), \mathrm{m}, \mathrm{n}=1,2, \cdots, N$, in the existence of anomaly $\Sigma$ can be represented as follows

$$
\mathrm{S}(\mathrm{m}, \mathrm{n}) \approx-\frac{k^{2}}{4 i \omega \mu} \int_{\Omega} \chi(\mathbf{r}) \mathbf{E}_{\mathrm{inc}}\left(\mathbf{r}_{\mathrm{n}}, \mathbf{r}\right) \mathbf{E}_{\mathrm{tot}}\left(\mathbf{r}, \mathbf{r}_{\mathrm{m}}\right) d \mathbf{r}
$$

where

$$
\chi(\mathbf{r})=\frac{\varepsilon(\mathbf{r})-\varepsilon_{\mathcal{B}}}{\varepsilon_{\mathcal{B}}}+i \frac{\sigma(\mathbf{r})-\sigma_{\mathcal{B}}}{\omega \varepsilon_{\mathcal{B}}} .
$$

Here, description of parameters are summarized in TABLE I.

TABLE I. DESCRIPTION OF PARAMETERS

\begin{tabular}{|c|c|}
\hline Parameter & Description (value) \\
\hline $\mathbf{r}_{\mathrm{n}}$ & Location of n-th antenna \\
\hline $\mathbf{r}$ & Search point in $\Omega$ \\
\hline$\varepsilon(\mathbf{r})$ & Permittivity at $\mathbf{r}$ \\
\hline$\sigma(\mathbf{r})$ & Conductivity at $\mathbf{r}$ \\
\hline$\varepsilon_{\mathcal{B}}$ & Background permittivity \\
\hline$\sigma_{\mathcal{B}}$ & Background conductivity \\
\hline$\mu$ & Background permeability $\left(4 \pi \cdot 10^{-7} \mathrm{H} / \mathrm{m}\right)$ \\
\hline$\omega$ & Angular frequency \\
\hline$k$ & Wavenumber \\
\hline $\mathbf{E}_{\text {inc }}\left(\mathbf{r}_{\mathrm{n}}, \mathbf{r}\right)$ & Incident field (z-component) \\
\hline $\mathbf{E}_{\text {tot }}\left(\mathbf{r}, \mathbf{r}_{\mathrm{m}}\right)$ & Total field (z-component) \\
\hline
\end{tabular}

Traditional MUSIC algorithm is based on the following scattering matrix:

$$
\mathbb{K}=\left[\begin{array}{ccccc}
\mathrm{S}(1,1) & \mathrm{S}(1,2) & \cdots & \mathrm{S}(1, N-1) & \mathrm{S}(1, N) \\
\mathrm{S}(2,1) & \mathrm{S}(2,2) & \cdots & \mathrm{S}(2, N-1) & \mathrm{S}(2, N) \\
\vdots & \vdots & \ddots & \vdots & \vdots \\
\mathrm{S}(N, 1) & \mathrm{S}(N, 2) & \cdots & \mathrm{S}(N, N-1) & \mathrm{S}(N, N)
\end{array}\right] .
$$

However, as we mentioned before, diagonal elements $S(n, n)$, $\mathrm{n}=1,2, \cdots \mathrm{N}$, are affected by the anomaly and antennas. Thus, we consider the following scattering matrix by eliminating diagonal elements and setting them as 0 such that 


$$
\mathbb{D}=\left[\begin{array}{ccccc}
0 & \mathrm{~S}(1,2) & \cdots & \mathrm{S}(1, N-1) & \mathrm{S}(1, N) \\
\mathrm{S}(2,1) & 0 & \cdots & \mathrm{S}(2, N-1) & \mathrm{S}(2, N) \\
\vdots & \vdots & \ddots & \vdots & \vdots \\
\mathrm{S}(N, 1) & \mathrm{S}(N, 2) & \cdots & \mathrm{S}(N, N-1) & 0
\end{array}\right] .
$$

The MUSIC algorithm is follows. First, let us perform the singular value decomposition

$$
\mathbb{D}=\mathbb{U S V}^{*}=\sum_{n=1}^{N} \tau_{n} \mathbf{U}_{n} \mathbf{V}_{n}^{*} \approx \sum_{n=1}^{M} \tau_{n} \mathbf{U}_{n} \mathbf{V}_{n}^{*}
$$

Then, $\left\{\mathbf{U}_{n}: n=1,2, \cdots, M\right\}$ spans signal subspace and $\left\{\mathbf{U}_{n}: n=M+1, M+2, \cdots, N\right\}$ spans noise subspace. With this, the projection operator onto the noise subspace can be introduced as follows

$$
\mathrm{P}_{\text {noise }}:=\mathbb{I}-\sum_{n=M+1}^{N} \mathbf{U}_{n} \mathbf{U}_{n}^{*}
$$

Now, let us introduce a test vector

$$
\mathbf{f}(\mathbf{r})=\left(\mathbf{E}_{\mathrm{inc}}\left(\mathbf{r}_{1}, \mathbf{r}\right), \mathbf{E}_{\mathrm{inc}}\left(\mathbf{r}_{2}, \mathbf{r}\right), \cdots, \mathbf{E}_{\mathrm{inc}}\left(\mathbf{r}_{\mathrm{n}}, \mathbf{r}\right)\right) \text {. }
$$

With this, the imaging function of MUSIC can be defined as

$$
\mathcal{F}(\mathbf{r})=\frac{1}{\left|\mathrm{P}_{\text {noise }} \mathbf{f}(\mathbf{r})\right|} .
$$

Then, the plot of imaging function $\mathcal{F}(\mathbf{r})$ is expected to exhibit peaks of large magnitude at $\mathbf{r} \in \Sigma$.

\section{Simulation Results}

We exhibit the simulation results for comparing the imaging performance with and without diagonal elements of scattering matrix. For this, we use $N=16$ dipole antennas and apply a frequency of $f=1 \mathrm{GHz}$. Every elements of scattering matries $\mathbb{K}$ and $\mathbb{D}$ were generated through the CST STUDIO SUITE with simulation configuration, refer to Figure 1.

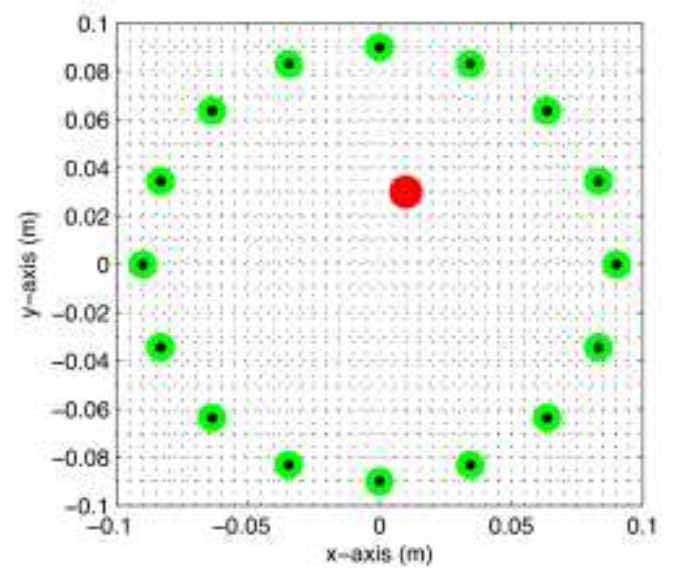

Figure 1. Illustration of simulation configuration. Red-colored circle is anomaly and green-colored circles are antennas.
Figures 2 and 3 show the distribution of singular values of $\mathbb{K}$ and $\mathbb{D}$, respectively, and map of $\mathcal{F}(\mathbf{r})$ (see [13] also). If one uses $\mathbb{K}$ for imaging, 5 left-singular vectors must be adopted for generating projection operator because the diagonal elements are affected by the anomaly and antennas. However, if one uses $\mathbb{D}$, there is only one nonzero singular value so that only the first left-singular vector is needed to generate the projection operator. Notice that based on several works $[6,8,11,12]$, total number of nonzero singular value is same as the total number of small anomalies. Thus, by eliminating diagonal elements, we can examine similar phenomenon.
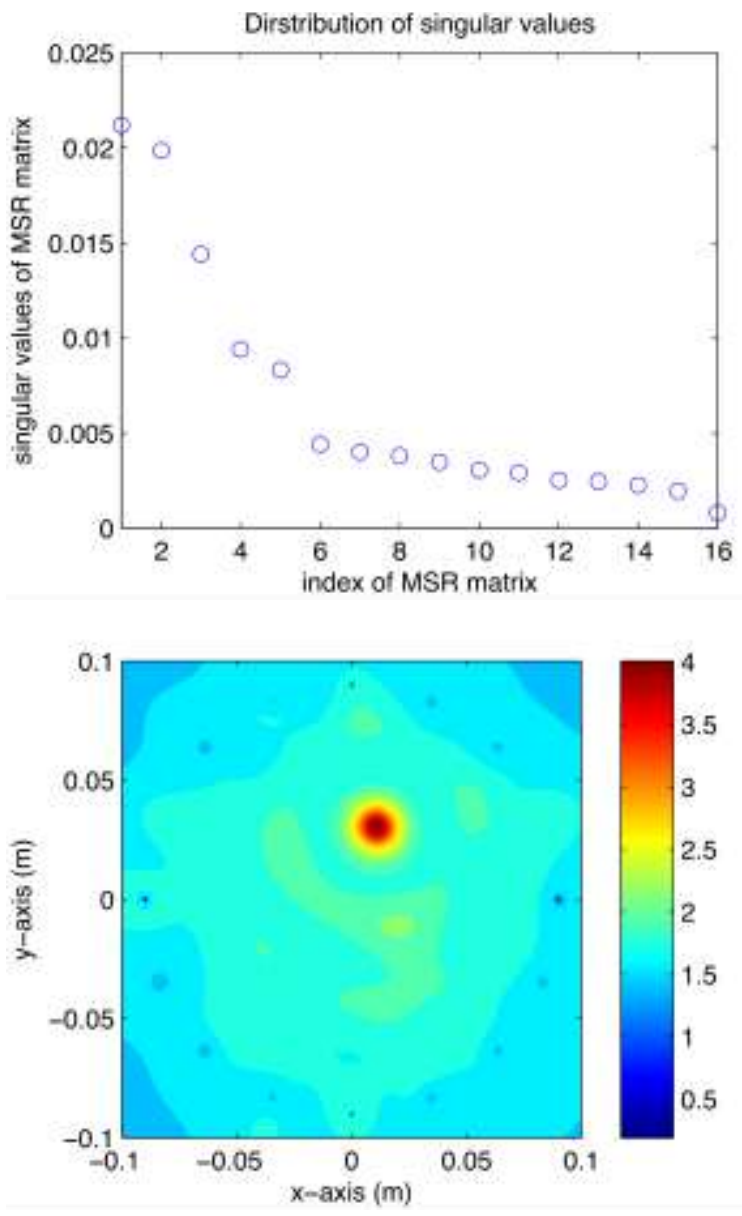

Figure 2. Distribution of nonzero singular values of $\mathbb{K}$ and map of $\mathcal{F}(\mathbf{r})$.

Now, let us compare the imaging performance. If one uses $\mathbb{K}$ or $\mathbb{D}$, there is no problem to identify the location and outline shape of anomaly via the map of $\mathcal{F}(\mathbf{r})$. However, there exists some unexpected artifacts in the map of $\mathcal{F}(\mathbf{r})$ with $\mathbb{K}$. In contrast, there is no artifacts in the map of $\mathcal{F}(\mathbf{r})$ with $\mathbb{D}$. Therefore, we can conclude that opposite to the traditional results, eliminating diagonal elements of scattering matrix will yield good result in microwave imaging. 
Proc. of the Eighth Intl. Conf. on Advances in Computing, Electronics and Communication - ACEC 2019

Copyright (C) Institute of Research Engineers and Doctors. All rights reserved.

ISBN: 978-1-63248-165-8 DOI : 10.15224/978-1-63248-165-8-14

\section{Conclusion}

Throughout simulation results, we examined that diagonal elements of scattering matrix significantly affect to the MUSIC-type imaging technique. Correspondingly, eliminating diagonal elements of scattering matrix yields better result than traditional approach. Establishment of mathematical theory will be the forthcoming work.

\section{Acknowledgment}

This research was supported by the Basic Science Research Program of the National Research Foundation of Korea (NRF) funded by the Ministry of Education (No. NRF2017R1D1A1A09000547).
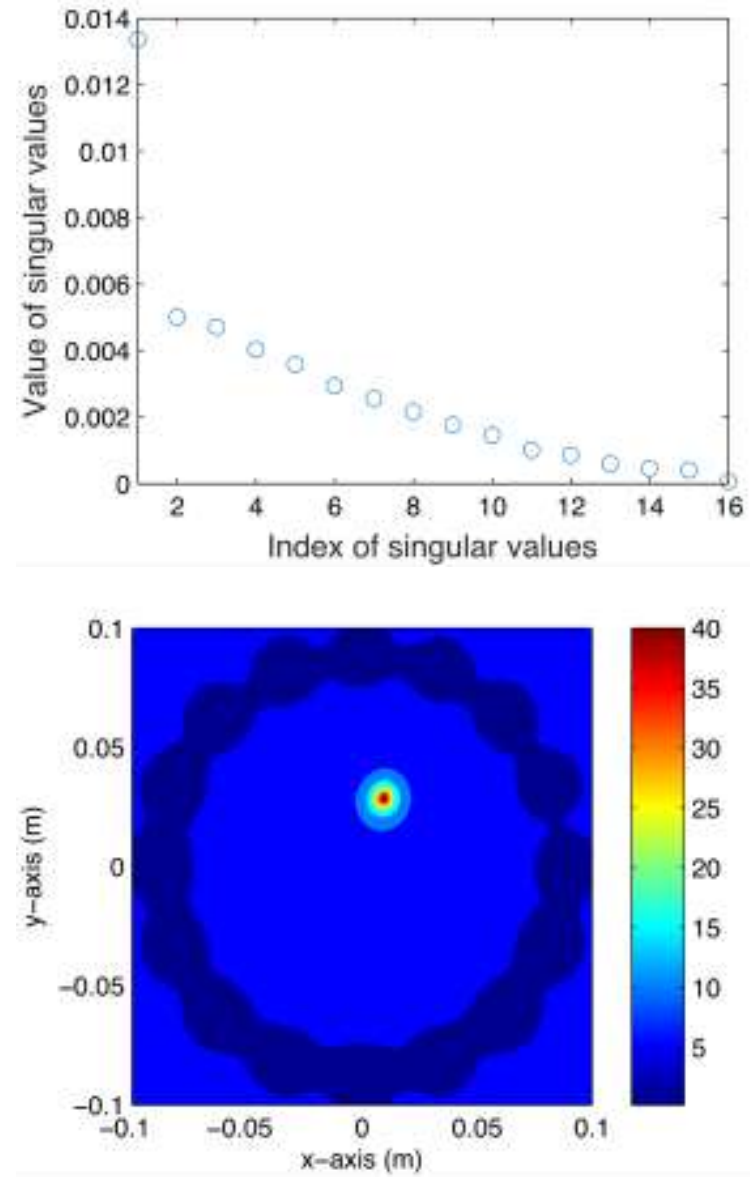

Figure 3. Distribution of nonzero singular values of $\mathbb{K}$ and map of $\mathcal{F}(\mathbf{r})$.

\section{References}

[1] M. Burger, "A level set method for inverse problems, " Inverse Problems, vol. 17, pp. 1327-1356, 2001.

[2] O. Dorn, E. L. Miller, and C. M. Rappaport, "A shape reconstruction method for electromagnetic tomography using adjoint fields and level sets, " Inverse Problems, vol. pp. 1119-1156, 2000.

[3] N. Irishina, O. Dorn, and M. Moscoso, "A level set evolution strategy in microwave imaging for early breast cancer detection," Comput. Math. Appl., vol. 56, pp. 607-618, 2008.
[4] G. Ventura, J. X. Xu, and T. Belytschko, "A vector level set method and new discontinuity approximations for crack growth by EFG," Int. J. Numer. Meth. Engng., vol. 54, pp. 923-944, 2002

[5] C. Y. Ahn, K. Jeon, and W.-K. Park, "Analysis of MUSIC-type imaging functional for single, thin electromagnetic inhomogeneity in limitedview inverse scattering problem," J. Comput. Phys., vol. 291, pp. 198217, 2015.

[6] H. Ammari, E. Iakovleva, D. Lesselier, and G. Perrusson, "MUSIC type electromagnetic imaging of a collection of small three-dimensional inclusions," SIAM J. Sci. Comput., vol. 29, pp. 674-709, 2007.

[7] X. Chen and Y. Zhong, "MUSIC electromagnetic imaging with enhanced resolution for small inclusions," Inverse Problems, vol. 25, ID 015008, 2009.

[8] M. Cheney, "The linear sampling method and the MUSIC algorithm," Inverse Problems, vol. 17, pp. 591-595, 2001.

[9] S. Hou, K. Sølna, and H. Zhao, "A direct imaging algorithm for extended targets," Inverse Problems, vol. 22, 1151-1178, 2006.

[10] E. Iakovleva, S. Gdoura, D. Lesselier, and G. Perrusson, "Multi-static response matrix of a 3D inclusion in half space and MUSIC imaging," IEEE Trans. Antennas Propag., vol. 55, pp. 2598-2609, 2007.

[11] Y.-D. Joh, Y. M. Kwon, W.-K. Park, "MUSIC-type imaging of perfectly conducting cracks in limited-view inverse scattering problems," Appl. Math. Comput., vol. 240, pp. 273-280, 2014.

[12] W.-K. Park, "Asymptotic properties of MUSIC-type imaging in twodimensional inverse scattering from thin electromagnetic inclusions," SIAM J. Appl. Math., vol. 75, pp. 209-228, 2015.

[13] W.-K. Park, H.P. Kim, K.-J. Lee, and S.-H. Son, "MUSIC algorithm for location searching of dielectric anomalies from S-parameters using microwave imaging,” J. Comput. Phys., vol. 348, pp. 259-270, 2017.

[14] B. Scholz, "Towards virtual electrical breast biopsy: space frequency MUSIC for trans-admittance data," IEEE Trans. Med. Imag., vol. 21, pp. 588-595, 2002.

[15] W.-K. Park, "Real-time microwave imaging of unknown anomalies via scattering matrix," Mech. Syst. Signal Proc., vol. 118, pp. 658-674, 2019.

[16] M. Haynes, J. Stang, and M. Moghaddam, "Real-time microwave imaging of differential temperature for thermal therapy monitoring," IEEE Trans. Biomed. Eng., vol. 61, pp. 1787-1797, 2014.

About Author (s):

Won-Kwang Park received his Ph. D. degree in Applied Mathematics from Ecole Polytechnique in 2009. After graduation, he joined the institute for mathematics and scientific computing, University of Graz as a post doctoral researcher in 2009. Currently, he is an associate professor of department of information security, cryptology, and mathematics in Kookmin University. His main research area includes Inverse scattering problem, microwave imaging, scientific computing, 